\title{
Foxl1 promotes liver repair following cholestatic injury in mice
}

\author{
Sara D Sackett ${ }^{1,3, *}$, Yan Gao ${ }^{2,3}$, Soona Shin ${ }^{1}$, Yonah B Esterson², Akivaga Tsingalia ${ }^{2}$, Reginald S Hurtt ${ }^{2}$, \\ Karrie Brondell ${ }^{1}$, Klaus $\mathrm{H}$ Kaestner ${ }^{1}$ and Linda E Greenbaum ${ }^{2}$
}

Cholangiocyte proliferation is one of the hallmarks of the response to cholestatic injury. We previously reported that the winged helix transcription factor Foxl1 is dramatically induced in cholangiocytes following bile duct ligation. In this study, we investigated the function of Foxl1 in the bile duct ligation model of cholestatic liver injury in Foxl1 ${ }^{-1-}$ and control mice. We found that Foxl1 $1^{-1}$ livers exhibit an increase in parenchymal necrosis, significantly impaired cholangiocyte and hepatocyte proliferation, and failure to expand bile ductular mass. Wnt3a and Wnt7b expression was decreased in the livers of Fox $11^{-1-}$ mice along with reduced expression of the $\beta$-catenin target gene Cyclin D1 in Foxl1 $1^{-1-}$ cholangiocytes. These results show that Foxl1 promotes liver repair after bile-duct-ligation-induced liver injury through activation of the canonical wnt $/ \beta$-catenin pathway.

Laboratory Investigation (2009) 89, 1387-1396; doi:10.1038/labinvest.2009.103; published online 19 October 2009

KEYWORDS: cholangiocyte; bile duct ligation; Wnt; $\beta$-catenin; Cyclin D1

The spectrum of cholangiopathies includes genetic and acquired diseases of the intrahepatic biliary tree. Damage to bile duct epithelial cells, or cholangiocytes by a variety of insults typically results initially in biliary hyperplasia. This so-called 'ductular reaction' is composed of reactive cholangiocytes that are thought to arise from hepatic progenitor cells located at the interface between the biliary tree and hepatocytic parenchyma initially restoring bile ductular mass (reviewed in Alvaro and Mancino ${ }^{1}$ ). These reactive cholangiocytes also express neural and mesenchymal markers not normally expressed in the healthy liver. In addition, growth factors, cytokines and angiogenic factors that act in both autocrine and paracrine manner are produced by both reactive cholangiocytes and nearby portal fibroblasts, hepatic stellate cells, endothelial cells and inflammatory cells to facilitate repair of biliary damage. When biliary injury becomes chronic, the failure to maintain an adequate cholangiocyte proliferative response combined with increased fibroblast and stellate cell collagen deposition ultimately results in a ductopenic state associated with portal fibrosis, which can progress to end-stage cirrhosis and liver failure.
Although a variety of mediators of cholangiocyte proliferation and cell death have been identified (see Refs. 1-4 and references contained therein) the characterization of the mechanisms of biliary repair remains incomplete.

We recently reported that Foxl1, a member of the forkhead box family of transcriptional regulators, is dramatically induced in cholangiocytes both in the bile duct ligation (BDL) model of obstructive cholestasis and in ductular cells in response to 3,5-diethoxycarbonyl-1,4-dihydrocollidine diet. $^{5}$ Moreover, genetic lineage tracing in these two models of ductular proliferation established that Foxll is expressed in cholangiocytes that represent a bipotential hepatic progenitor cell population. Although nothing is known about the function of Foxll in the liver, in the gut, Foxll is downstream of the hedgehog pathway and is a mediator of the wnt/ $\beta$ catenin pathway, two signaling pathways that have been linked to cholangiocyte viability and proliferation, respectively. ${ }^{6-11}$ Taken together, these findings led us to investigate whether Foxl1 is important for cholangiocyte viability and/or proliferation following bile duct injury. Foxl1 $1^{-1-}$ mice do not exhibit any baseline liver abnormalities indicating that Foxl1

\footnotetext{
'Department of Genetics, Division of Gastroenterology, University of Pennsylvania School of Medicine, Philadelphia, PA, USA and ${ }^{2}$ Department of Medicine, University of Pennsylvania School of Medicine, Philadelphia, PA, USA

Correspondence: Dr LE Greenbaum, MD, Department of Cancer Biology and Medicine, Thomas Jefferson University, 233 S 10 th Street, 306 BLSB, Philadelphia, PA 19107, USA.

E-mail: linda.greenbaum@jefferson.edu

${ }^{3}$ These authors contributed equally to this work.

${ }^{*}$ Current Address: Medizinische Klinik II, Universitatsklinikum Aachen, Aachen, Germany.

Received 29 May 2009; revised 10 August 2009; accepted 17 August 2009
} 
is dispensable for liver development. ${ }^{10,12}$ The livers of these mice, however, develop increasing necrosis at late time points after BDL, associated with reduced cholangiocyte and hepatocyte proliferation, and decreased expansion of bile ductular mass. The reduced expression of Wnt $3 \mathrm{a}, \mathrm{Wnt} 7 \mathrm{~b}$ and the Wnt target gene Cyclin D1 in Foxl1 mutants places Foxll as an upstream mediator of $\beta$-catenin-induced cholangiocyte proliferation.

\section{MATERIALS AND METHODS}

\section{Mice and Experimental Protocols}

The derivation of Foxl1 ${ }^{-l-}$ mice and wild-type controls $\left(\right.$ Foxl1 ${ }^{+/+}$) used for bile duct ligations has been described previously. ${ }^{12}$ The mice were maintained on a C57Bl/6J background. Genotyping was performed using the following primers and polymerase chain reaction (PCR) conditions: Foxl1 5'-CGCGTCGAGCCCCCGCAGAAG; and Foxl1 3'-CC CCTTCTCACGTG-GCACCTTC; and LacZ CGCCATTCGCC ATTCAGGCTGC. PCRs were performed under the following conditions: $95^{\circ} \mathrm{C}, 5 \mathrm{~min}, 1$ cycle; $95^{\circ} \mathrm{C}, 45 \mathrm{~s} ; 67^{\circ} \mathrm{C}, 45 \mathrm{~s} ; 72^{\circ} \mathrm{C}$, $90 \mathrm{~s}$ for 35 cycles; followed by $72^{\circ} \mathrm{C}, 5 \mathrm{~min}$ for 1 cycle. Foxl1 ${ }^{-/-}$ mice are smaller than littermate controls at birth as a result of delayed maturation of the intestinal epithelium. ${ }^{12}$ By $10-16$ weeks, however, the weight of surviving mice is not different from controls (mean weight at time of surgery was $26 \mathrm{~g}$ for Foxl $1^{+1+}$ mice and $24 \mathrm{~g}$ for Foxl $1^{-1-}$ mice). Only males were used for the study. Foxl1 ${ }^{-1-}$ and Foxl1 ${ }^{+/+}$mice underwent BDL or sham surgery between 12 and 24 weeks of age. Shamoperated mice underwent ventral laparotomy with bile duct dissection only, as described previously. ${ }^{5}$ Mice were allowed to recover and either 5 or 14 days postoperatively, were anesthetized with isofluorane and blood was collected through the portal vein for serological analysis. The livers were then removed and partitioned for multiple purposes. Parts of each lobe were placed in $4 \%$ paraformaldehyde (PFA) and processed for histology and immunohistochemistry or frozen for subsequent RNA extraction and gene expression analysis. All protocols were approved by the institutional animal care and use committee of the University of Pennsylvania.

\section{RNA Isolation and Quantitative Real-Time Polymerase Chain Reaction}

Total RNA was extracted from liver samples using the Totally RNA kit (Ambion, Applied Biosystems, Foster City, CA, USA). Liver RNA was reverse transcribed using oligo dT priming and SuperScript II reverse transcriptase (Invitrogen, Carlsbad, CA, USA). Primer sequences were selected using the Primer3 program. ${ }^{13}$ Quantitative real-time PCRs were performed using SYBR Green QPCR Master Mix (Invitrogen) on an Mx3000 or Mx4000 PCR cycler (Stratagene, Agilent Technologies, Santa Clara, CA, USA). Reactions were performed in triplicate with reference dye normalization and median $C_{\mathrm{t}}$ values were used in the analyses. Primer sequences were as follows:
Wnt3a (F): 5'-GTGATGTCCATGCCCCAAAT-3' ${ }^{\prime}$, Wnt3a (R): 5'-GGAATCAGATGGGTCCTGAAAC-3'; Wnt7a (F): 5' CTGGTGAAACTCAGATGCCTGT-3', Wnt7a (R): 5'-GGGA AGAAATCAGCCATACAGC-3'; Wnt7b (F): $5^{\prime}$-TCCCCTGTC TGTCATGTCTCTT-3', Wnt7b (R): $5^{\prime}$-CTGTTTCAAGCAGA AGGAGGAG-3'; Wnt10a (F): 5'-GTGATAGGCAATGCACT GAAGC-3', Wnt10a (R): 5'-CGGCCATTTCTGCCTTTTA G-3'; TATA binding protein (F): 5'-CCCCTTGTACCCTTCA CCAAT-3', TATA binding protein (R): 5'-GAAGCTGCGGTA CAATTCCAG-3'.

\section{Serum Biochemistry Analysis}

The serum levels of alanine aminotransferase, asparate aminotransferease (AST) and alkaline phosphatase, and total bilirubin were analyzed by Ani Lytics Inc. (Gaithersburg, MD, USA).

\section{Quantification of Parenchymal Necrosis}

Following overnight fixation in 4\% PFA, tissues were paraffin-embedded and $6 \mu \mathrm{m}$ tissue sections were obtained. Trichrome-stained tissue sections were used to analyze the areas of necrosis using IPLab software (BioVision, Becton-Dickinson, Exton, PA, USA) in a masked manner. The percentage of necrosis was evaluated by measuring necrotic areas/total area of tissue minus the area of the vascular lumen.

\section{Quantification of Cholangiocyte Area}

For immunodetection of CK19, paraffin-embedded sections were rehydrated in graded alcohols and then subjected to antigen retrieval by microwaving for $14 \mathrm{~min}$ in $10 \mathrm{mM}$ citric acid monohydrate buffer ( $\mathrm{pH}$ 6.0). Rat anti-CK19 (1:100; TROMA III developed by R Kemler was obtained from the Developmental Studies Hybridoma Bank under the auspices of the NICHD and maintained by the University of Iowa, Ames, IA, USA) was diluted and immunodetection of CK19 was performed using the Vector $\mathrm{ABC}$ Elite kit according to the manufacturer's instructions (Vector Laboratories, Burlingame, CA, USA).

To calculate the cholangiocyte area, we imaged 14 fields containing CK19-positive cells at $\times 10$ magnification using the segmentation function of IPLab software and were used to calculate the ratio of the CK19-positive area to the total area.

\section{Quantification of Hepatocyte Proliferation}

Paraffin-embedded tissue sections were incubated with antiKi67 (1:500 dilution; Vector Laboratories) antibody and immunodetection was carried out using the $\mathrm{ABC}$ Vector Elite kit (Vector Laboratories) according to the manufacturer's instructions. The number of positively stained hepatocytes was calculated in ten $\times 20$ fields for each sample and expressed as a percentage of the total hepatocytes. 


\section{Quantification of Cholangiocyte Proliferation}

Paraffin-embedded tissue sections were rehydrated, subjected to antigen retrieval with citric acid as described above, blocked with Protein-Blocking Reagent (Beckman Coulter, Fullerton, CA, USA) and incubated with rat anti-CK19 (1:100 dilution; Hybridoma Development) antibody and mouse anti-PCNA (1:9000 dilution; catalog no. M0879; Dako, Carpinteria, CA, USA) overnight at $4{ }^{\circ} \mathrm{C}$. Sections were then incubated with donkey $\mathrm{Cy} 3$-anti-mouse (1:600 dilution) and donkey Cy2-anti-rat (1:600 dilution; Jackson ImmunoResearch Laboratories, West Grove, PA, USA). Slides were washed in PBS, dried and coverslip was applied with aqueous mounting medium. Ten $\times 20$ microscope fields containing bile ducts were examined for each sample and the ratio of PCNA-positive cholangiocytes/total cholangiocytes was calculated.

\section{TUNEL Assay}

Paraffin-embedded sections were heated at $60^{\circ} \mathrm{C}$ for $10 \mathrm{~min}$ and rehydrated in graded alcohols. Sections were pretreated with proteinase $\mathrm{K}$ (Roche, Indianapolis, IN, USA) in PBS for $15 \mathrm{~min}$ at room temperature. Endogenous peroxidases were quench with $30 \% \mathrm{H}_{2} \mathrm{O}_{2}$ in $\mathrm{ddH}_{2} \mathrm{O}$ for $15 \mathrm{~min}$ at room temperature followed by a $15 \mathrm{~min}$ room temperature block with 3\% bovine serum albumin in PBS. For apoptosis detection the ApopTag Apoptosis Detection kit (Millipore, Temecula, CA, USA) was used. The Vector ABC Elite kit detection method was used according to manufacturer's instructions (Vector Laboratories). Counterstain was then applied with Gill no. 2 hematoxylin (Sigma-Aldrich, St Louis, MO, USA).

\section{Statistical Analysis}

Student's $t$-tests with equal variance and two-tailed distribution were used to determine the significance of differences between two groups (Excel statistical analysis software; Microsoft, Redmond, VA, USA). A P-value of 0.05 or lower was considered statistically significant. Results where indicated are expressed as mean \pm s.e.

\section{RESULTS}

To assess acute and chronic differences in response to bile duct injury, we subjected control (Foxl1 ${ }^{+/+}$) and Foxl1 ${ }^{-1-}$ mice to either sham surgery or bile duct ligation and livers were harvested 5 and 14 days after the procedure. There was no difference in mortality between the control and BDL cohorts for 5- or 14-day survival; however, we were unable to extend our analysis to later time points due to nearly $100 \%$ mortality in the Foxl1 $1^{-1-}$ mice beyond 14 days after BDL. Serum AST was elevated to a greater degree in the Foxl1 ${ }^{-/-}$ mice than in controls 14 days after BDL. $(874 \pm 140 \mathrm{IU} / \mathrm{ml}$ controls vs $1320 \pm 121 \mathrm{IU} / \mathrm{ml}$ Foxl1 ${ }^{-1-}$ mice, $\left.P<0.05\right)$ suggesting greater hepatic injury in the Foxl1 mutants. Histological examination of hematoxylin-and-eosin- as well as trichrome-stained liver sections indicated that although necrosis was similar in control and Foxl1 ${ }^{-1-}$ livers 5 days after BDL, hepatic necrosis was more extensive in the Foxl1 ${ }^{-1-}$ livers at the 14-day post-BDL time point (Figure 1).

Hepatocellular necrosis following bile duct ligation represents biliary infarcts that occur as the result of toxic bile acid extravasation from the bile ducts. The percentage of CK19-positive cells co-expressing Foxl1 increases dramatically after BDL. ${ }^{5}$ Therefore we hypothesized that the increased parenchymal necrosis in the Foxl1 ${ }^{-1-}$ livers postBDL model is likely to be the result of an inadequate proliferative response of the subpopulation of ductular cells that normally express Foxll. To investigate whether ductular cell expansion was impaired in the absence of Foxll, we measured ductular area in control and Foxl1 ${ }^{-1-}$ livers by quantifying the CK19-stained fraction of the total parenchymal area. Bile ductular area increased initially to a similar extent in control and Foxl1 ${ }^{-1-}$ livers 5 days after BDL. Although we detected a progressive increase in ductular area at the 14-day post-BDL time point in the control livers, ductular area did not increase in the Foxl1 ${ }^{-1-}$ livers (Figure 2).

Expansion of bile ductular area in response to obstruction is thought to represent a balance between cholangiocyte proliferation and cell death. We therefore sought to investigate whether there was reduced cholangiocyte proliferation and/or increased apoptosis in the Foxl1 ${ }^{-1-}$ livers relative to the controls. PCNA staining was significantly reduced in Foxl1 ${ }^{-1-}$ cholangiocytes 5 days after BDL (Figure 3) indicating that cholangiocyte proliferation was impaired. There was no difference in cholangiocyte apoptosis at either 5- or 14-day post-BDL time points between Foxl1 ${ }^{-1-}$ and control livers (Supplementary Figure S1). Taken together, these findings indicate that the reduced cholangiocyte area in the Foxl1 $1^{-1-}$ livers is the result of decreased cholangiocyte proliferation rather than increased cell death.

In the bile duct ligation model, hepatocyte proliferation is a compensatory reparative response to bile-acid-induced hepatocyte injury. In control livers, hepatocyte proliferation was maximal 5 days after BDL, similar to what has been reported previously. ${ }^{14}$ Unexpectedly, although hepatic necrosis was similar in Foxl1 ${ }^{-/-}$and control livers 5 days after BDL, hepatocyte proliferation was significantly reduced in Foxl1 $1^{-1-}$ livers at this time point (Figure 4). Hepatocyte proliferation was sustained 14 days after BDL in the Foxl1 ${ }^{-1-}$ livers, consistent with a compensatory response to the increased parenchymal necrosis. Although we hypothesized that in the setting of increased injury, fibrosis would also be increased in the Foxl1 ${ }^{-1-}$ livers after BDL, there was no difference in fibrosis between Foxl1 ${ }^{-l-}$ and control livers based on our examination of trichrome- and Sirius-redstained slides or hydroxyproline assay (not shown) in the 5- and 14-day BDL livers. Foxl1 is not expressed in either hepatic stellate cells or portal fibroblasts. ${ }^{5}$

We therefore considered whether fibrosis might in fact be more extensive in the Foxl1 $1^{-1-}$ livers at later time points, but not yet apparent at the 14-day time point when repair was 

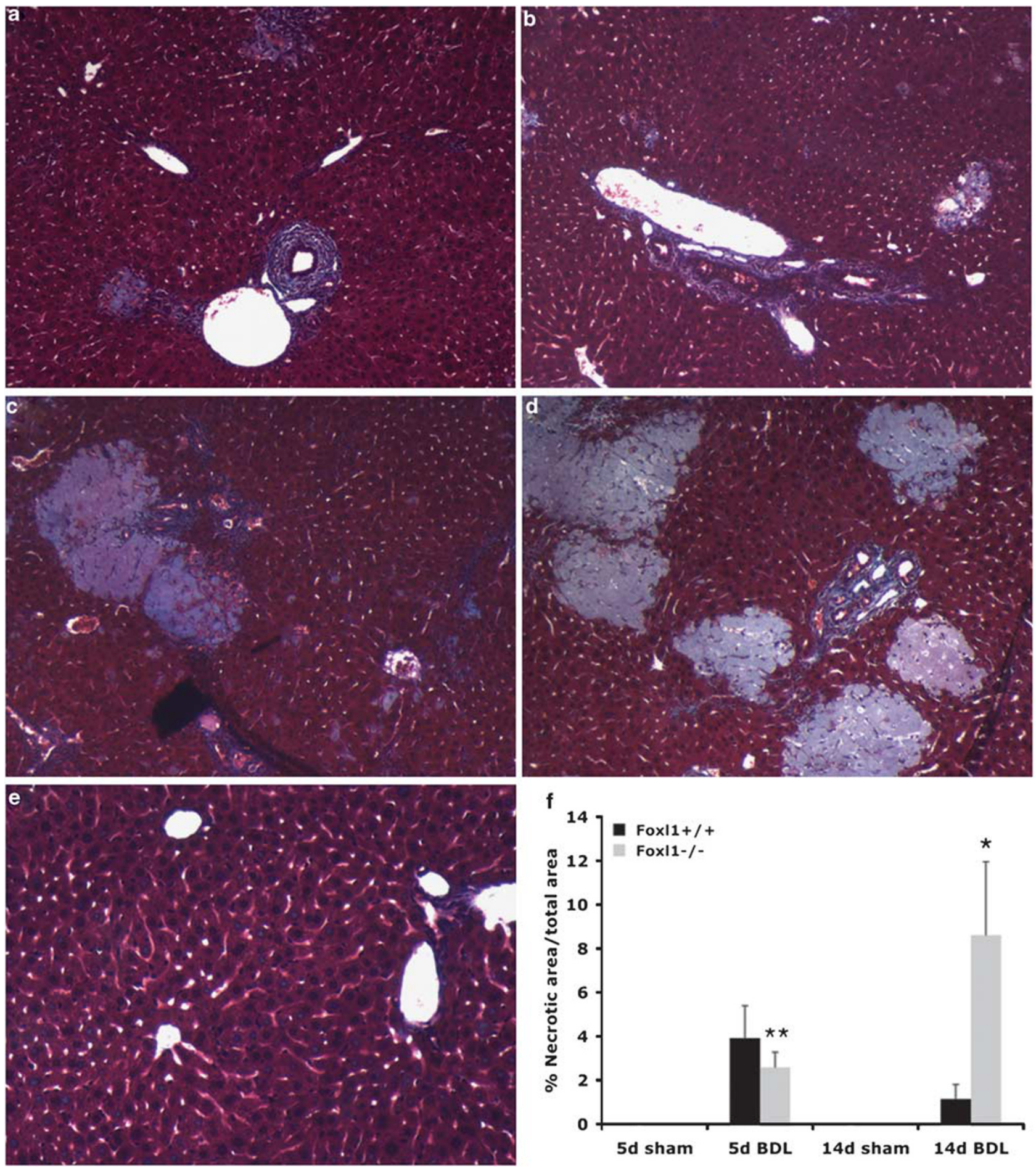

Figure 1 Foxl1 $1^{-1-}$ livers exhibit more extensive parenchymal necrosis than controls following bile duct ligation (BDL). Representative trichrome-stained liver sections from Foxl1 $1^{+/+}$5-day post-BDL (a), Foxl1 ${ }^{-/-}$-day post-BDL (b), Foxl1 ${ }^{+/+}$14-day post-BDL (c), Foxl1 ${ }^{-/-}$14-day post-BDL (d) and Foxl1 ${ }^{+/+}$ 5-day sham-operated mice (e). (f) Quantification of necrotic parenchyma as a percentage of total parenchymal area. ${ }^{*} P=0.05$ relative to Foxl1 ${ }^{+/+} 14$-day $\mathrm{BDL},{ }^{* *} P<0.05$ relative to Foxl1 ${ }^{-/-} 5$-day sham. $N=2-7$ for each time point; $\times 10$ magnification for a-d; $\times 20$ magnification shown for $\mathbf{e}$.

still incomplete. Unfortunately, we were unable to extend our analysis to later time points to address this possibility due to the nearly $100 \%$ mortality in the Foxl1 ${ }^{-/-}$mice beyond 14 days after BDL.
Foxl1 regulates the wnt/ $\beta$-catenin pathway in the gut. ${ }^{10}$ In the liver, expression of canonical wnt ligands is increased in proliferating cholangiocytes and ductular progenitor cells and these cells proliferate in response to wnt ligands. ${ }^{15-17} \mathrm{We}$ 

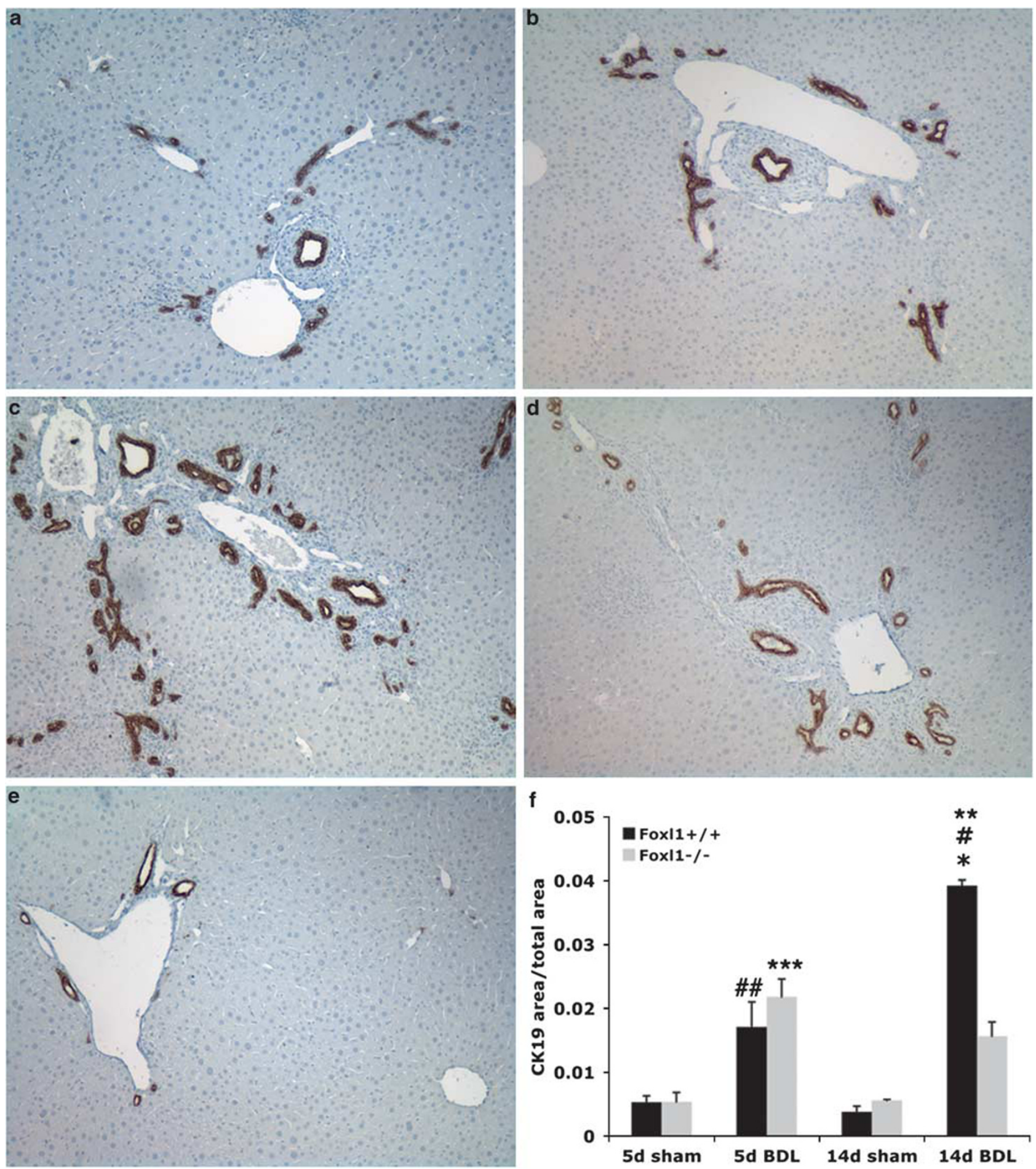

Figure 2 Cholangiocyte area expansion is blunted in Foxl1 $1^{-/-}$livers following bile duct ligation (BDL). Representative CK19-stained liver sections from Foxl1 ${ }^{+/+}$5-day post-BDL (a), Foxl1 ${ }^{-/-} 5$-day post-BDL (b), Foxl1 ${ }^{+/+}$14-day post-BDL (c), Foxl1 $1^{-1-}$ 14-day post-BDL (d) and Foxl1 ${ }^{+/+} 5$-day sham-operated mice (e). (f) Quantification of CK19 area/total area. ${ }^{*} P<0.05$ relative to Foxl1 ${ }^{-1-}$ 14-day BDL, ${ }^{\#} P=0.01$ relative to Foxl1 ${ }^{+/+} 5$-day BDL, ${ }^{\# \#} P<0.05$ relative to Foxl1 ${ }^{+/+} 5$-day sham, ${ }^{* * P}<0.0001$ relative to Foxl1 ${ }^{+/+} 14$-day sham, ${ }^{* *} P<0.05$ to relative to Foxl1 ${ }^{-1-} 5$-day sham. $N=2-4$ for each time point; $\times 10$ magnification for all images.

therefore investigated whether reduced wnt expression was responsible for the decreased cholangiocyte proliferation in the Foxl1 ${ }^{-1-}$ BDL livers. Quantitative real-time PCR analysis indicated that the expression of both Wnt3a and Wnt7b was reduced 5 days after BDL in Foxl1 ${ }^{-1-}$ livers (Figure 5). Several other canonical wnts including Wnts $7 \mathrm{a}$ and 10a were 


\section{PCNA CK19}
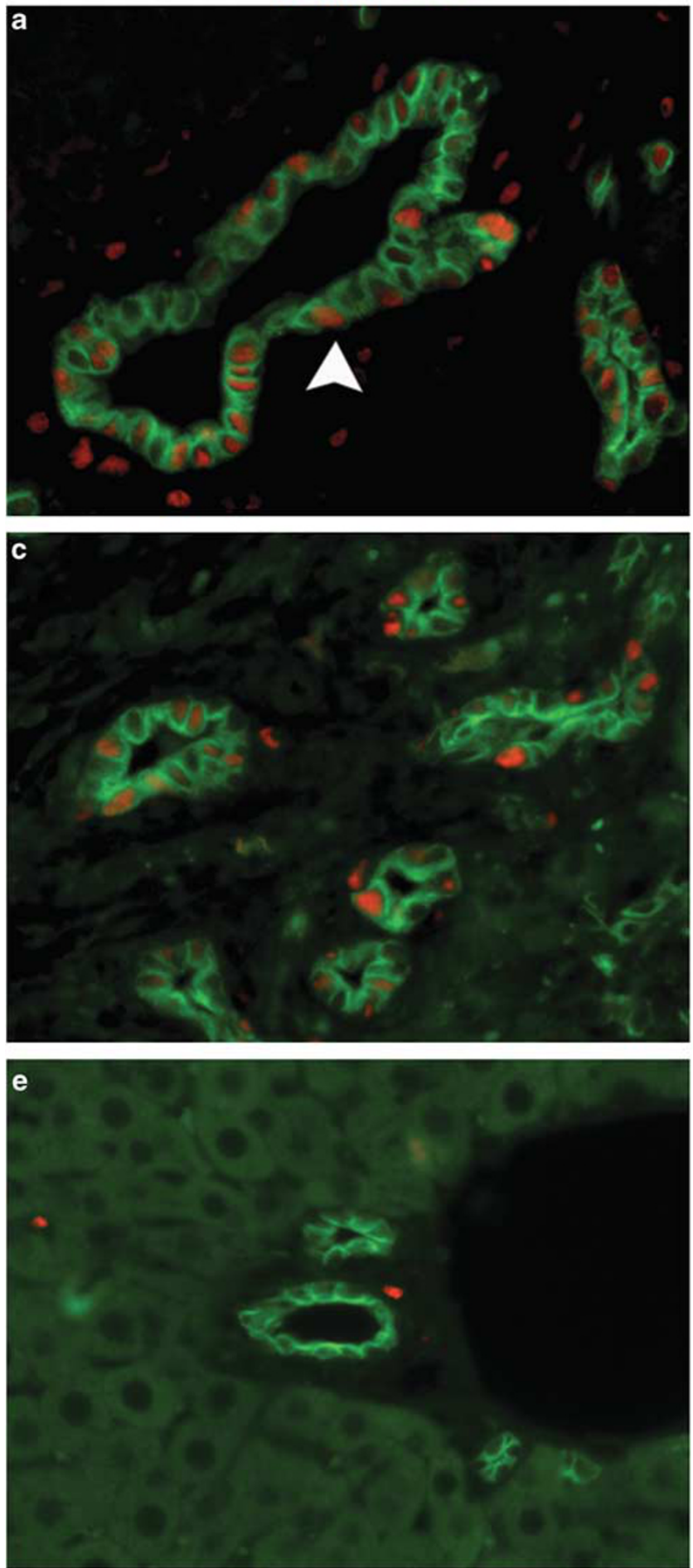
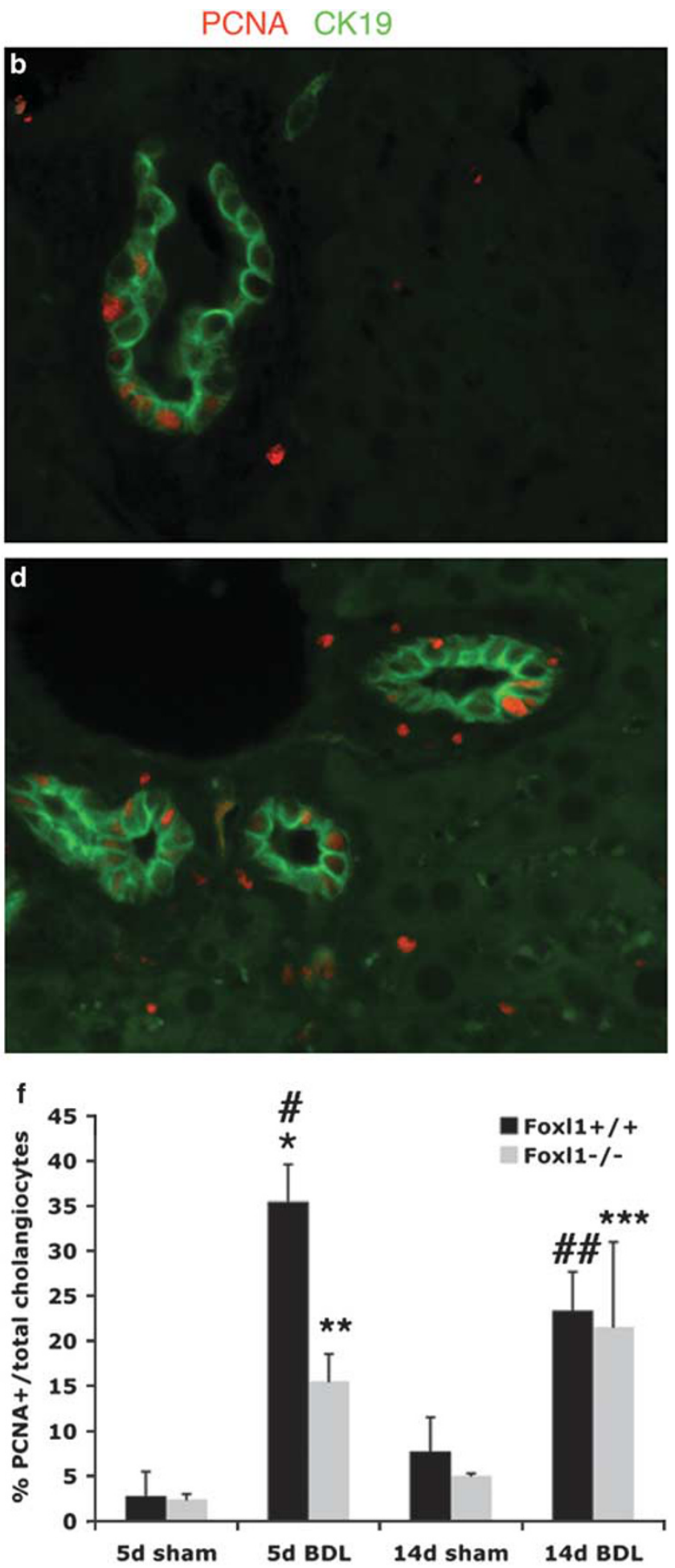

Figure 3 Cholangiocyte proliferation is reduced in Foxl1 ${ }^{-1-}$ livers in bile duct-ligated livers. Cholangiocyte proliferation was quantified by simultaneous PCNA and CK19 staining in livers of 5- and 14 -day post-BDL Foxl1 $1^{+/+}$, Foxl1 $1^{-1-}$ and sham-operated mice. Representative liver tissue sections from Foxl1 ${ }^{+/+}$5-day post-BDL (a), Foxl1 ${ }^{-/-}$5-day post-BDL (b), Foxl1 ${ }^{+/+}$14-day post-BDL (c), Foxl1 ${ }^{-1-}$ 14-day post-BDL mice (d) and 5-day Foxl1 ${ }^{+/+}$ sham-operated mice $(\mathbf{e})$. Magnification $(\times 40)$ for all images. Arrowhead indicates representative PCNA/CK19-positive cholangiocyte. Quantification of percent PCNA/CK19-stained cells was performed on $\times 20$ magnification images at indicated time points (f). ${ }^{*} P<0.01$ relative to Foxl1 ${ }^{-1-} 5$-day BDL, ${ }^{* *} P<0.05$ relative to Foxl1 ${ }^{-/-} 5$-day sham, ${ }^{\#} P<0.05$ relative to Foxl1 ${ }^{+/+} 5$-day sham, ${ }^{\# \#} P<0.05$ relative to Foxl1 ${ }^{+/+} 5$-day $B D L$, ${ }^{* * *} P<0.05$ relative to Foxl1 ${ }^{-/-}$14-day sham. $N=3-7$ for each time point. 

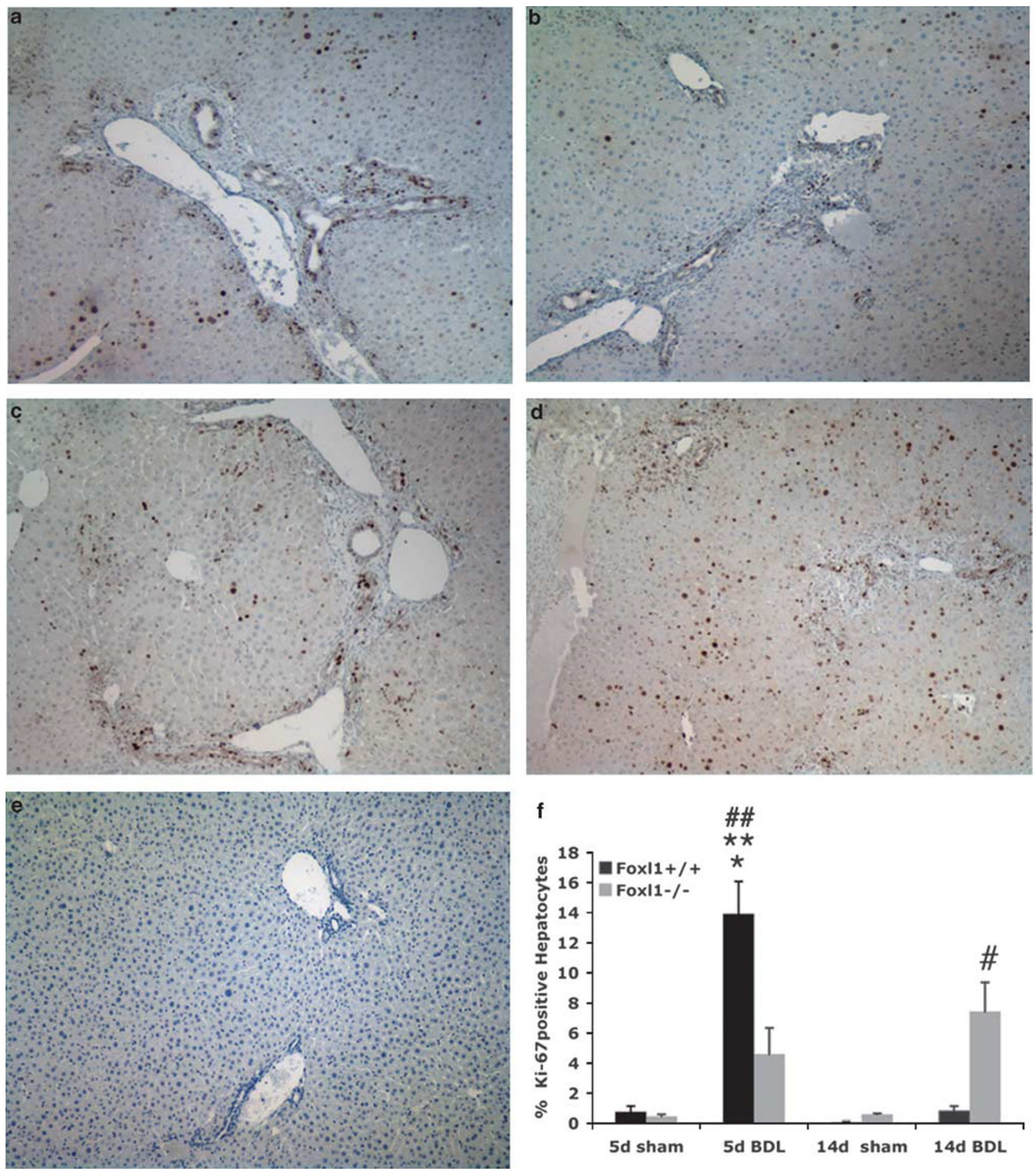

Figure 4 Hepatocyte proliferation is delayed in Foxl1 $1^{-1-}$ livers following cholestatic liver injury. Hepatocyte proliferation was quantified by Ki67 staining in livers 5- and 14-day post-BDL and sham-operated Foxl1 ${ }^{+/+}$and Foxl1 ${ }^{-1-}$ livers. Representative sections from Foxl1 ${ }^{+/+}$5-day post-BDL (a), Foxl1 ${ }^{-1-}$-day post-BDL (b), Foxl1 ${ }^{+/+}$14-day post-BDL (c), Foxl1 ${ }^{-1-}$ 14-day post-BDL (d) and Foxl1 ${ }^{+/+}$14-day sham-operated mice (e). (f) Quantification of percent Ki67positive hepatocytes at indicated time points after BDL. ${ }^{\star} P<0.05$ relative to 5 -day Foxl $1^{-1-} \mathrm{BDL},{ }^{* *} P=0.001$ relative to Foxl1 ${ }^{+/+} 14$-day $\mathrm{BDL}$, ${ }^{\#} P<0.05$ relative to 14 -day Foxl1 ${ }^{+/+} \mathrm{BDL},{ }^{\# \#} P<0.05$ relative to Foxl1 ${ }^{+/+}$5-day sham. $N=4-7$ samples for each time point; $\times 10$ magnification for all images.

significantly induced 5 and/or 14 days after BDL. However, expression was not different between Foxl1 $1^{-1-}$ and Foxl1 ${ }^{+/+}$ mice (Figure 5). Activation of the canonical wnt $/ \beta$-catenin signaling pathway in the liver by wnt ligands results in nuclear translocation of dephosphorylated $\beta$-catenin and activation of wnt target genes including Cyclin D1. ${ }^{18}$ To 

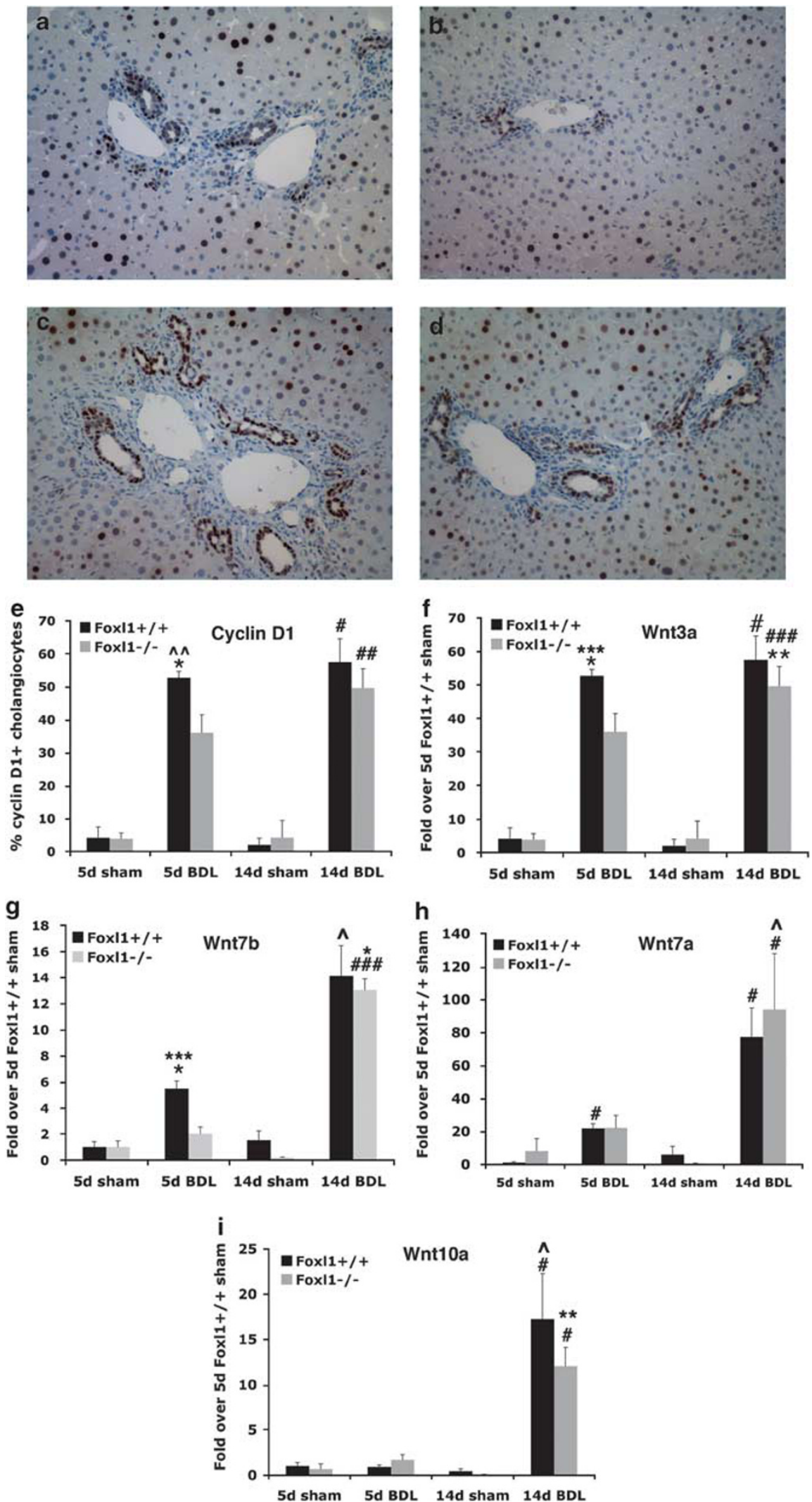
determine whether reduced wnt expression in the Foxl1 ${ }^{-1-}$ livers resulted in impaired $\beta$-catenin signaling in cholangiocytes, we investigated whether the expression of the wnt target gene Cyclin D1 was reduced in Foxl1 ${ }^{-1-}$ cholangiocytes. Cyclin D1 expression was significantly decreased in Foxl1 ${ }^{-1-}$ cholangiocytes 5 days after BDL corresponding to the time at which Wnt3a and Wnt7b expression was reduced. Taken together, these findings indicate that Foxl1 is required for wnt $/ \beta$-catenin activation of cholangiocyte proliferation following cholestatic liver injury.

\section{DISCUSSION}

Cholangiocyte proliferation is a critical component of the repair process that occurs following damage to the bile ducts during the course of cholestatic liver diseases. Because the equilibrium between cholangiocyte proliferation and cell death ultimately tips in favor of the latter resulting in ductopenia in end-stage cholestatic liver diseases, identification of novel therapeutic approaches to enhance cholangiocyte proliferation may reduce morbidity in chronic cholestatic liver disorders. Here we have provided evidence that the winged helix transcription factor Foxl1 is important for the proliferative response of cholangiocytes in the BDL model of cholestatic liver injury. We show further that this proliferative response is mediated through activation of the cell-cycle protein, cyclin D1, an established downstream target of the canonical wnt $/ \beta$-catenin signaling pathway. Although we did not detect differences in cholangiocyte apoptosis, an accurate assessment of apoptotic cholangiocytes is particularly challenging, due to the transitory presence of apoptotic cholangiocytes and sloughing of apoptotic cells into the biliary lumen. Therefore, we cannot exclude the possibility that in addition to the defect in cholangiocyte proliferation, Foxl1 ${ }^{-1-}$ cholangiocytes may also be more susceptible to either apoptotic and/or necrotic cell death. There are no reliable antibodies for Foxll. Therefore, we cannot definitively confirm that the ductular cells in which proliferation is reduced reflect the same population of cells that activate Foxll, ie, the facultative progenitor cells. To address this question, future plans will include crossing the Foxl1 ${ }^{-1-}$ and Foxl1 ${ }^{+1+}$ mice with Foxl1Cre and the YFP reporter mouse strain so that we can quantify differences in proliferation and apoptosis that occur specifically in the Foxll progenitor cell compartment.
Reactive cholangiocytes secrete growth factors and immune mediators that signal hepatocytes, immune cells and other nonparenchymal cell populations in the liver through paracrine interactions. These signals not only enhance cholangiocyte proliferation but also cause increased inflammation and fibrosis (Alvaro et $a l^{4}$ and references therein). The acquisition of these enhanced properties by reactive cholangiocytes is consistent with our observation that Foxll is only expressed in conditions associated with cholangiocyte injury. Diehl and colleagues have recently reported that the sonic hedgehog pathway is reactivated in response to bile duct ligation in rodents ${ }^{7-9}$ and that hedgehog ligands secreted by hepatic stellate cells enhance cholangiocyte viability and motility. ${ }^{7}$ Foxl1 is a downstream target of the sonic hedgehog signaling pathway in the gut ${ }^{6}$ and it is reasonable to speculate that Foxl1 may represent an important hedgehog target responsible for the effects of sonic hedgehog on cholangiocyte function during cholestatic liver injury. Previously, Monga et $a l^{16}$ established that recombinant Wnt3a treatment of cholangiocytes in culture is associated with enhanced cell survival and increased proliferation. The present study extends these observations to the in vivo setting. The reduced expression of Wnt3a and Wnt7b in Foxl1 ${ }^{-1-}$ livers and decreased cholangiocyte expression of the $\mathrm{wnt} / \beta$-catenin target cyclin D1 in Foxl1 ${ }^{-1-}$ cholangiocytes provide strong support for Foxl1 activation of cholangiocyte proliferation through the wnt $/ \beta$-catenin pathway following cholangiocyte injury. Unexpectedly, although hepatic necrosis was similar in Foxl $1^{-1-}$ and control livers 5 days after BDL, hepatocyte proliferation was significantly reduced in $\mathrm{Foxll}^{-1-}$ livers. Several groups have reported that hepatocyte proliferation in the partial hepatectomy model is dependent upon activation of the wnt $/ \beta$-catenin signaling pathway, although the source of wnt ligands has not identified. ${ }^{19,20}$ We propose that Foxl1 activation of Wnt3a and/or Wnt7b in cholangiocytes regulates not only cholangiocyte proliferation through an autocrine mechanism, but may also stimulate hepatocyte proliferation through paracrine activation of the $\beta$-catenin signaling pathway. We did not detect differences in fibrosis in the Foxll $^{-1-}$ livers after BDL despite more severe parenchymal necrosis. The nearly $100 \%$ mortality of Foxl1 ${ }^{-l-}$ at time points later than 14 days after BDL did not allow us to directly assess whether fibrotic reaction was delayed but ultimately greater in the Foxl1 ${ }^{-1-}$ livers relative to controls.

Figure 5 Expression of the Wnt target gene Cyclin D1 and canonical Wnts $3 \mathrm{a}$ and $7 \mathrm{~b}$ is reduced in Foxl1 ${ }^{-1-}$ cholangiocytes following bile duct ligation (BDL)-induced liver injury. Cyclin D1 expression in cholangiocytes was quantified in 5 - and 14-day post-BDL Foxl1 ${ }^{+/+}$, Foxl1 ${ }^{-1-}$ and sham-operated livers Representative liver tissue sections from Foxl1 ${ }^{+/+}$5-day post-BDL (a), Foxl1 ${ }^{-1-}$ 5-day post-BDL (b), Foxl1 ${ }^{+/+}$14-day post-BDL (c) and Foxl1 ${ }^{-1-} 14-$ day post-BDL mice (d). Quantification of percent Cyclin-D1-positive cholangiocyte in ten $\times 20$ fields at indicated time points after BDL (e). Wnt3a (f) and Wnt7b (g) expression is significantly reduced in Foxl1 ${ }^{-1-}$ livers 5 days after BDL. Wnt7a (h) and Wnt 10a (i) are significantly induced after BDL but their expression is not reduced in Foxl1 ${ }^{-1-}$ livers. ${ }^{*} P<0.05$ relative to 5 -day Foxl1 ${ }^{-1-} \mathrm{BDL},{ }^{\#} P<0.05$ relative to 5 -day Foxl1 ${ }^{+/+}$sham, $\wedge \wedge P<0.001$ relative to 5 -day Foxl1 ${ }^{+/+}$ sham, ${ }^{\#} P<0.05$ relative to Fox $11^{+/+} 14$-day sham, ${ }^{\# \#} P<0.001$ relative to Foxl1 ${ }^{-/-} 14$-day sham, ${ }^{* * *} P<0.05$ relative to Foxl1 ${ }^{+/+} 5$-day sham, ${ }^{\# \# \#} P<0.05$ relative to Foxl1 ${ }^{-1-}$ 14-day sham, $\wedge P<0.05$ relative to Foxl1 ${ }^{+/+} 5$-day BDL, ${ }^{* * P}<0.01$ relative to Foxl1 ${ }^{-1-} 5$-day BDL. $N=3-7$ samples per time point; $\times 20$ magnification for all images. 
Alternatively, it is also possible that the increased necrosis detected in the Foxl1 ${ }^{-1-}$ livers 14 days after BDL reflects the combined effects of reduced ductular cell expansion and defective activation or reduced viability of the hepatic stellate cell and/or portal fibroblasts. The increased mortality observed in the Foxl1 ${ }^{-I-}$ mice with longer periods after BDL suggests that activation of compensatory pathways was not sufficient for repair in this model, in which survival is highly dependent on a rapid cholangiocyte proliferative response. It will be informative to investigate whether Foxll expression is activated during early stages of cholangiopathies in patients and decreases at later stages when ductopenia and increased fibrosis are present. Such information could help identify the critical stages at which the therapeutics to increase Foxll expression and/or wnt activity would prove beneficial. Future studies will also investigate the contribution of the sonic hedgehog pathway in activation of Foxll, as well as the basis for Foxll activation of the wnt $/ \beta$-catenin pathway during cholestatic liver injury.

Supplementary Information accompanies the paper on the Laboratory Investigation website (http://www.laboratoryinvestigation.org)

\section{ACKNOWLEDGEMENTS}

We thank Beth Helmbrecht, Tim Luo and Li-ya Yu for expert technical assistance, and Gary Swain and members of the University of Pennsylvania Center for Digestive Disease Morphology Core for their histology service. This study was supported by University of Pennsylvania Institute for Regenerative Medicine (to LEG and KHK), NIH Center Grant P-30-DK050306.

\section{DISCLOSURE/CONFLICT OF INTEREST}

There are no financial interests to disclose.

1. Alvaro $D$, Mancino MG. New insights on the molecular and cell biology of human cholangiopathies. Mol Aspects Med 2008;29:50-57.

2. Lazaridis KN, Strazzabosco M, Larusso NF. The cholangiopathies: disorders of biliary epithelia. Gastroenterology 2004;127:1565-1577.

3. Glaser SS, Gaudio E, Miller T, et al. Cholangiocyte proliferation and liver fibrosis. Expert Rev Mol Med 2009;11:e7.

4. Alvaro D, Mancino MG, Glaser S, et al. Proliferating cholangiocytes: a neuroendocrine compartment in the diseased liver. Gastroenterology 2007;132:415-431.
5. Sackett SD, Li Z, Hurtt R, et al. Foxl1 is a marker of bipotential hepatic progenitor cells in mice. Hepatology 2009;49:920-929.

6. Madison BB, McKenna LB, Dolson D, et al. FoxF1 and FoxL1 link hedgehog signaling and the control of epithelial proliferation in the developing stomach and intestine. J Biol Chem 200927;284: 5936-5944.

7. Omenetti $A$, Popov $Y$, Jung $Y$, et al. The hedgehog pathway regulates remodelling responses to biliary obstruction in rats. Gut 2008;57: $1275-1282$.

8. Omenetti $A$, Porrello $A$, Jung $Y$, et al. Hedgehog signaling regulates epithelial-mesenchymal transition during biliary fibrosis in rodents and humans. J Clin Invest 2008;118:3331-3342.

9. Omenetti A, Yang L, Li YX, et al. Hedgehog-mediated mesenchymalepithelial interactions modulate hepatic response to bile duct ligation. Lab Invest 2007;87:499-514.

10. Perreault N, Katz JP, Sackett SD, et al. Foxl1 controls the Wnt/betacatenin pathway by modulating the expression of proteoglycans in the gut. J Biol Chem 2001;276:43328-43333.

11. Perreault N, Sackett SD, Katz JP, et al. Foxl1 is a mesenchymal modifier of Min in carcinogenesis of stomach and colon. Genes Dev 2005;19:311-315.

12. Kaestner $\mathrm{KH}$, Silberg DG, Traber PG, et al. The mesenchymal winged helix transcription factor Fkh6 is required for the control of gastrointestinal proliferation and differentiation. Genes Dev 1997;11:1583-1595.

13. Rozen S, Skaletsky H. Primer3 on the WWW for general users and for biological programmers. Methods Mol Biol 2000;132: $365-386$.

14. Georgiev $\mathrm{P}$, Jochum W, Heinrich $\mathrm{S}$, et al. Characterization of timerelated changes after experimental bile duct ligation. $\mathrm{Br} J$ Surg 2008;95:646-656.

15. Monga SP, Monga HK, Tan X, et al. Beta-catenin antisense studies in embryonic liver cultures: role in proliferation, apoptosis, and lineage specification. Gastroenterology 2003;124:202-216.

16. Hussain SZ, Sneddon T, Tan X, et al. Wnt impacts growth and differentiation in ex vivo liver development. Exp Cell Res 2004;292: 157-169.

17. Hu $\mathrm{M}$, Kurobe $\mathrm{M}$, Jeong $\mathrm{YJ}$, et al. Wnt/beta-catenin signaling in murine hepatic transit amplifying progenitor cells. Gastroenterology 2007;133:1579-1591.

18. Tan X, Yuan $Y$, Zeng $G$, et al. Beta-catenin deletion in hepatoblasts disrupts hepatic morphogenesis and survival during mouse development. Hepatology 2008;47:1667-1679.

19. Tan X, Behari J, Cieply B, et al. Conditional deletion of beta-catenin reveals its role in liver growth and regeneration. Gastroenterology 2006;131:1561-1572.

20. Goessling W, North TE, Lord AM, et al. APC mutant zebrafish uncover a changing temporal requirement for wnt signaling in liver development. Dev Biol 2008;320:161-174. 